\title{
Neural Signatures of Memory Encoding in Schizophrenia Are Modulated by Antipsychotic Treatment
}

\author{
Demet Gurler ${ }^{a}$ David Matthew White ${ }^{a}$ Nina Vanessa Kraguljac ${ }^{a}$ \\ Lawrence Ver Hoef $^{\mathrm{b}}$ Clinton Martin ${ }^{\mathrm{a}}$ Blake Tennant ${ }^{\mathrm{a}} \quad$ Adrienne Carol Lahti $^{\mathrm{a}}$ \\ a Department of Psychiatry and Behavioral Neurobiology, University of Alabama at Birmingham, Birmingham, AL, \\ USA; ${ }^{b}$ Department of Neurology, University of Alabama at Birmingham, Birmingham, AL, USA
}

\section{Keywords}

Encoding $\cdot$ Retrieval $\cdot$ Hippocampus $\cdot$ Default mode network · Glutamate $\cdot$ Antipsychotic treatment $\cdot$ Functional magnetic resonance imaging $\cdot$ Magnetic resonance spectroscopy

\begin{abstract}
There is no pharmacological treatment to remediate cognitive impairment in schizophrenia (SZ). It is imperative to characterize underlying pathologies of memory processing in order to effectively develop new treatments. In this longitudinal study, we combined functional magnetic resonance imaging during a memory encoding task with proton MR spectroscopy to measure hippocampal glutamate + glutamine (GIx). Seventeen SZ were scanned while unmedicated and after 6 weeks of treatment with risperidone and compared to a group of matched healthy controls (HC) scanned 6 weeks apart. Unmedicated patients showed reduced blood oxygen level dependent (BOLD) response in several regions, including the hippocampus, and greater BOLD response in regions of the default mode network (DMN) during correct memory encoding. Post hoc contrasts from significant group by time interactions indicated reduced hippocampal BOLD
\end{abstract}

response at baseline with subsequent increase following treatment. Hippocampal Glx was not different between groups at baseline, but at week 6, hippocampal Glx was significantly lower in SZ compared to HC. Finally, in unmedicated SZ, higher hippocampal Glx predicted less deactivation of the BOLD response in regions of the DMN. Using 2 brain imaging modalities allowed us to concurrently investigate different mechanisms involved in memory encoding dysfunction in SZ. Hippocampal pathology during memory encoding stems from decreased hippocampal recruitment and faulty deactivation of the DMN, and hippocampal recruitment during encoding can be modulated by antipsychotic treatment. High Glx in unmedicated patients predicted less deactivation of the DMN; these results suggest a mechanism by which faulty DMN deactivation, a hallmark of pathological findings in SZ, is achieved.

(c) 2020 S. Karger AG, Basel

\section{Introduction}

Approximately $75-85 \%$ of schizophrenia (SZ) patients show impairments in cognition with selective deficits in learning and memory [1]. Importantly, impairments in

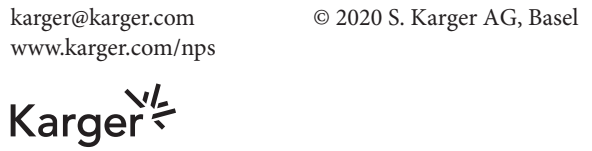

Adrienne C. Lahti, MD

University of Alabama at Birmingham Sparks Center, Room 501

1720 7th Ave. S, Birmingham, AL 35233 (USA)

alahti@uabmc.edu 
episodic memory are not exclusively explained by overall impairments in IQ or executive functioning [2]. Recently, combination of brain imaging techniques has become a common practice in research to take advantage of crossinformation and improve upon identification of pathological signatures of diseases [3]. Neural correlates of episodic memory processing in patients with SZ can be studied using appropriate behavioral paradigms along with functional magnetic resonance imaging (fMRI) [4, 5]. Proton magnetic resonance spectroscopy (MRS) allows for in vivo measurement of brain metabolites such as glutamate, an amino acid involved in excitatory neurotransmission [6] and metabolism [7, 8]. Given the role of glutamate as the main excitatory neurotransmitter involved in cognition [9] and the role of the hippocampus in learning and memory processes, measurement of glutamate in the hippocampus could provide insights into the pathology of learning and memory impairments in SZ. Importantly, several groups have now reported elevated levels of glutamate in medication-naïve or unmedicated SZ in different brain regions [10,11], including in the hippocampus [12].

Cumulative evidence indicates that memory deficits in $\mathrm{SZ}$ might be related to impairments in intentional encoding $[13,14]$. Previous studies have shown a positive relationship between encoding success and hippocampus blood oxygen level dependent (BOLD) response, pointing to the role of the hippocampus in the integration of information from different cortical regions [15-17]. It has been shown that hippocampus and parahippocampal gyrus activation during encoding is predictive of subsequent retrieval success $[18,19]$. Several neuroimaging studies reported BOLD signal abnormalities in the hippocampus and parahippocampal gyrus both in SZ [20] and in unaffected relatives [21-24]. However, these studies have produced discrepant findings, demonstrating both increased and decreased BOLD response in SZ during memory tasks [25].

Suppression of default mode network (DMN) regions during memory encoding plays an important role in achieving optimum memory performance [26]. The DMN is suppressed during cognitive demands but engaged during rest [27]. In SZ, hyperactivation of DMN during cognitive tasks has been extensively reported [28, 29]. Consistent with the role of glutamate in neuroenergetics [30], Hu et al. [31] reported a positive relationship between high glutamate concentration in the posterior $\mathrm{DMN}$ and reduced $\mathrm{DMN}$ deactivation during a working memory task. In addition, Kapogiannis et al. [32] identified a relationship between posterior DMN glutamate (posteromedial cortex) and the intrinsic functional connectivity of the DMN. Two studies have now identified a relationship between anterior cingulate cortex glutamate and the BOLD response in the posterior DMN in healthy controls (HC), and this relationship was found to be opposite in SZ [33,34]. There is a need to clarify the neurochemistry of DMN hyperactivation in SZ.

The vast majority of imaging studies of memory function have enrolled medicated SZ; however, it has been shown that antipsychotic medications have modulatory effects on brain function during rest $[35,36]$, and during cognitive tasks [37]. Therefore, it is unclear to what extent these findings are related to antipsychotic treatment rather than intrinsic characteristics of the illness. In addition, although the critical role of antipsychotic medications for symptom relief, such as hallucinations or delusions, is well established, their benefits on cognitive functions are more controversial [38]. However, studies that included large numbers of subjects such as CATIE [39] and EUFEST [40] have shown that treatment with a wide variety of both first- and second-generation antipsychotics is associated with moderate improvements on cognitive tests. Relevant to our aims, recent studies have reported that antipsychotic medications decrease glutamate levels [41-43].

The purpose of the current study was to examine encoding-related BOLD response and resting state hippocampal glutamate levels in SZ using a longitudinal design (before, while unmedicated, and after 6 weeks of antipsychotic treatment) to compare $\mathrm{HC}$ and $\mathrm{SZ}$ response without the effect of medication as a confounding factor and investigate the effect of medication on brain responses in SZ. We hypothesized that in SZ we would replicate findings of reduced BOLD response in regions previously found associated with memory processing, and increased BOLD response in regions of the DMN, as well as changes as a result of treatment. Based on prior findings, we hypothesized that hippocampal glutamate levels would be elevated before and reduced after treatment. In addition, we explored the relationship between hippocampal glutamate and BOLD response in the DMN before and after treatment.

\section{Materials and Methods}

\section{Participants}

Subjects with SZ and schizoaffective disorder (SZ) were recruited from the outpatient psychiatry clinics and emergency room at the University of Alabama at Birmingham to participate in the study based on being off antipsychotic medication for at least 10 
days (medication was not discontinued to meet this criteria). Among them, 17 patients completed both encode and retrieval sessions of the memory paradigm at 2 time points (Baseline/unmedicated and week 6). Seventeen HC subjects, without personal or family history in a first-degree relative of significant DSM-IV-TR Axis I disorders were recruited using advertisement in flyers and the university's newspaper. Exclusion criteria were major medical conditions, substance abuse or dependence (except for nicotine) within 6 months of imaging, previous head injury with loss of consciousness for $>2 \mathrm{~min}$, and pregnancy. The Institutional Review Board of the University of Alabama at Birmingham provided approval for the study, and all subjects gave written informed consent prior to participating.

Diagnoses were established using subjects' medical records and the Diagnostic Interview for Genetic Studies [44]. General cognitive function for each subject was characterized by the Repeatable Battery for the Assessment of Neuropsychological Status [45].

Patients were scanned while unmedicated, and then entered into a 6-week trial with risperidone (flexible dosing regimen), at the end of which they received a second scan. Symptom severity was assessed with the Brief Psychiatric Rating Scale [46] and its positive and negative subscales. Medication compliance was monitored by pill counts. HC were scanned twice at a 6-week interval. Prior to each scanning session, all subjects underwent a urine drug screen.

\section{Task Design and Behavioral Analysis}

The episodic memory task (see in $[4,47]$ for details) consisted of an intentional encoding phase, followed by a recognition memory phase after a 15-min delay. To maximize retrieval performance, a deep encoding paradigm utilizing an animacy decision was used. During the encoding task, participants saw a series of 60 words, presented one at a time for $300 \mathrm{~ms}$ followed by a fixation screen. A 2-s prestimulus cue (“Alive?") indicated that the participant had to answer by button press whether the upcoming word was alive or not alive. Following a $15 \mathrm{~min}$ interval, participants performed the retrieval task, where they saw 60 words, including 30 words previously seen (old words) and 30 new words, presented one at a time for 300 ms. A 2-s warning stimulus ("Ready?") indicated that the participant had to answer by button press whether the upcoming word was "old" or "new." Depending on participants' responses, these items were classified as hits, misses, correct rejections, and false alarms. An IFIS-SA system (In Vivo Corp., Orlando, FL, USA) running E-Prime software (version 1.2; Psychology Software Tools, Inc., Pittsburgh, PA, USA) controlled stimulus delivery and recorded responses and reaction times (RT).

A word was considered encoded correctly if it was successfully retrieved in the subsequent retrieval session. Task engagement was calculated using button presses during encoding trials. We used d-prime (d'), calculated based on the information during retrieval sessions, as the primary memory performance measure. $d$ ' is a measure of sensitivity by calculating the distance between signal and noise in standard deviation units ( $\mathrm{d}^{\prime}=\mathrm{z}$ [HITS] $-\mathrm{z}$ [FALSE ALARMS]) [48].

\section{Imaging Parameters}

All imaging data were acquired on a 3T head-only scanner (Siemens Allegra, Erlangen, Germany), equipped with a circularly polarized transmit/receive head coil. fMRI data were acquired using a gradient recalled echo-planar imaging sequence (repetition time/ echo time $[\mathrm{TR} / \mathrm{TE}] 2,100 / 30 \mathrm{~ms}$, flip angle $=70^{\circ}$, field of view $=$ $24 \times 24 \mathrm{~cm}^{2}, 64 \times 64$ matrix, $4 \mathrm{~mm}$ slice thickness, $1 \mathrm{~mm}$ gap, 26 axial slices). A high-resolution structural scan was acquired using a T1-weighted magnetization prepared rapid acquisition gradientecho sequence (TR/TE/inversion time 2,300/ 3.93/ 1,100 ms, flip angle $=12^{\circ}, 256 \times 256$ matrix, $1 \mathrm{~mm}$ isotropic voxels) .

A series of sagittal, coronal, and axial T1-weighted anatomical scans serving as MRS-localizers were acquired for spectroscopic voxel placement. Slices were aligned to the anatomical midline to control for head tilt. To facilitate voxel placement, the axial images were obtained along the long axis of the hippocampus, as viewed from the sagittal images. The voxel was placed in the left hippocampus such that the amount of gray matter was maximized (voxel size $2.7 \times 1.5 \times 1 \mathrm{~cm}^{3}$ ). Manual shimming was performed to optimize field homogeneity across the voxel, and chemical shift selective pulses were used to suppress the water signal. Spectra were acquired using the point-resolved spectroscopy sequence (TR/TE $=2,000 / 80 \mathrm{~ms}$ to optimize the glutamate signal [49] and minimize macromolecule contribution; $1,200 \mathrm{~Hz}$ spectral bandwidth; 1,024 points; 640 averages).

\section{Statistical Analyses}

Behavior and Demographics

Analyses were conducted using SPSS 20 (IBM SPSS Inc., Chicago, IL, USA). Group comparisons were performed using chisquare or analysis of variance, as appropriate. Analyses of response on encoding trials, reaction time (RT) for correct trials, percentage of correctly encoded words, and d' values were analyzed using linear mixed models comparing fixed effects of group (HC vs. SZ), time (unmedicated vs. week 6), and interactions. Post hoc analyses were performed where appropriate with Bonferroni corrections.

MRS Analysis

After removing the residual water peak, the MRS data were quantified in the time domain using the AMARES algorithm [50] in jMRUI (version 3.0). Prior knowledge derived from in vitro and in vivo metabolite spectra was included in the model [51], which consisted of peaks for $\mathrm{N}$-acetyl-aspartate, choline, creatine $(\mathrm{Cr})$, and 3 peaks for glutamate + glutamine (Glx). Amplitude, line width, and chemical shift were optimized for each peak. CramerRao lower bounds [52] were calculated for each peak. Exclusion criteria were Cramer-Rao lower bounds $>25 \%$. No data were excluded on the basis of these criteria. Glx were quantified with respect to Cr. MRS data were missing for $1 \mathrm{SZ}$, both at baseline and at week 6 , and for $2 \mathrm{HC}$ at baseline and $3 \mathrm{HC}$ at week 6. Differences between groups were tested using independent sample $t$ test at baseline and at week 6 . The alpha level was set at.05.

\section{fMRI Analysis}

Data analyses were implemented in SPM12 running in MATLAB (version R2013b). Preprocessing of the fMRI data included slice timing correction, realignment and reslicing to the mean functional volume, artifact/motion correction (movement $>1$ $\mathrm{mm}$ ) using ArtRepair, coregistration to the structural scan, and normalization to Montreal Neurological Institute (MNI) space using DARTEL [53] with 6-mm FWHM Gaussian kernel smoothing. Participants were excluded from further analyses if $33 \%$ or more of their data were repaired during artifact and motion correction.

The subject-level statistical analysis consisted of an event-related GLM with the following regressors: encode main effect, encode 
Table 1. Demographics and clinical variables

\begin{tabular}{|c|c|c|c|}
\hline Characteristics & $\mathrm{HC}(n=17)$ & $\mathrm{SZ}(n=17)$ & $p$ value \\
\hline Age, years & $31.77(8.6)$ & $31.24(8.96)$ & 0.861 \\
\hline Gender, male, \% & 71 & 82 & $0.409^{*}$ \\
\hline $\mathrm{SES}^{\mathrm{a}}$ & $6.00(4.55)$ & $8.42(5.55)$ & $0.692^{*}$ \\
\hline Smoking ${ }^{\mathrm{b}}$ & $0.32(.51)$ & $0.63(.48)$ & 0.08 \\
\hline RBANS total ${ }^{\mathrm{c}}$ & $91.29(10.49)$ & $71.71(12.08)$ & 0.000 \\
\hline BPRS total ${ }^{\mathrm{d}}$ & NA & $48(10.84)$ & NA \\
\hline BPRS positive & NA & $9(2.03)$ & NA \\
\hline BPRS negative & NA & $7.24(2.43)$ & NA \\
\hline
\end{tabular}

Values are presented as mean (SD).

a SES, ranks determined from diagnostic Interview for Genetic Studies (1-18 scale); higher rank (lower numerical value) corresponds to higher socioeconomic status. ${ }^{\mathrm{b}}$ Calculated using estimates based on packs per day. ${ }^{\mathrm{c}}$ Repeatable Battery for the RBANS; d BPRS, scored on a 1-7 scale; positive subscale (conceptual disorganization, hallucinatory behavior, and unusual thought content); negative subscale (emotional withdrawal, motor retardation, and blunted affect). $p$ values based on independent sample $t$ test. * Based on chi-square test.

HC, healthy control; SZ, patient with schizophrenia; SES, socioeconomic status; RBANS, Assessment of Neuropsychological Status; BPRS, Brief Psychiatric Rating Scale; NA, not applicable.

correct, and encode incorrect trials. A word was considered correctly encoded if it was successfully retrieved in the subsequent retrieval session. All events were modeled using a canonical hemodynamic response function, and data were high-pass filtered (cutoff $=256 \mathrm{~s}$ ). At the group level, statistical parametric maps of the BOLD signal during encoding were generated, and comparisons between groups were conducted using 2 -sample $t$ tests. For multiple comparison correction, the cluster size threshold was defined within SPM12, based on Gaussian random field theory, as the number of contiguous voxels with $p<0.05$ (uncorrected) in order to accept the false discovery rate of 0.05 [54].

To identify differences between groups specific to the DMN, we built a combined region of interest (ROI), inclusive of the medial frontal gyrus, posterior cingulate gyrus, precuneus, hippocampi, and inferior parietal gyri, using automated anatomical labeling atlas within the Wake Forest University Pick Atlas version 2.4 [55]. The anatomical correlates of the DMN mask were based on Buckner et al. [56]. Small-volume correction $p<0.05$ was used to correct for multiple comparisons.

To evaluate the effect of medication, we conducted a full factorial analysis, identifying brain regions sensitive to the interaction of group $\times$ time during encode correct trials. The subject- and parameter estimates of encode correct trials were entered into a random effect analysis using SPM's "full factorial model." Factors were time (baseline/unmedicated, week 6) and group (HC, SZ). For this whole brain analysis, the cluster size threshold for multiple comparisons was defined by Monte Carlo simulations using a voxel level threshold at $p=0.05$ with 1,000 simulations. For illustration purposes, the signal was extracted from significant regions using REX (CIBSR Stanford University, CA, USA) with a $6 \mathrm{~mm}$ ROI, and

Memory Encoding in SZ the extracted first eigenvariate signal was then plotted at each time point and for each group. In addition, to explore the relationship between regions where significant group $\times$ time interactions were identified and memory performance, the extracted first eigenvariate was plotted against each participant's associated d' prime value.

To explore the relationship between DMN BOLD and hippocampal Glx, the first eigenvariate of the "encode correct" contrast in the DMN ROI was extracted using REX, and we performed a bivariate correlation between the extracted data and hippocampal Glx measures using SPSS. The relationships between Glx and BOLD were analyzed by Pearson correlation and were compared using Fisher's $r$ to $\mathrm{Z}$ transform.

\section{Results}

\section{Behavior and Hippocampal Glutamate}

The groups were well matched in terms of age, gender, parental socioeconomic status, and smoking (Table 1).

Both $\mathrm{HC}$ and $\mathrm{SZ}$ responded on most encoding trials without significant differences between groups $\left(F_{1,32}=\right.$ $2.778 ; p=0.105)$, time $\left(F_{1,32}=0.001 ; p=0.98\right)$, or groupby-time interaction $\left(F_{1,32}=0.001 ; p=0.98\right)$. Mean RTs were longer for SZ compared to HC (HC, 1,100 ms; SZ, $\left.1,459 \mathrm{~ms} ; F_{1,29}=11.141 ; p=0.002\right)$ but a significant effect of time, or group-by-time interaction (HC baseline, 1,103 ms, week 6, 1,096 ms; SZ baseline, 1,530 ms, week 6, 1,389 $\mathrm{ms} ; F_{1,29}=2.294 ; p=0.141$ ), was not observed. There was a significant difference between groups in the percentage of correctly encoded words (HC, 85\%; SZ, 67\%; $F_{1,32}=$ $11.141 ; p=0.002)$ without any significant difference in time, or group-by-time interaction (HC baseline, $86 \%$, week 6, 84\%; SZ baseline, $66 \%$, week $6,67 \% ; F_{1,32}=0.306$; $p=0.584)$.

Comparison of d' values across group and time revealed significant main effects for group (HC, 2.1; SZ, $1.44 ; F_{1,32}=7.02 ; p=0.012$ ) but not time, or group-bytime interaction (HC baseline, 2.14, week 6, 2.07; SZ baseline, 1.43 , week $6,1.44 ; F_{1,32}=0.152 ; p=0.699$ ).

Hippocampal Glx levels did not significantly differ between groups at baseline, but at week 6, HC hippocampal Glx levels were significantly higher than SZ (baseline, $t=$ $0.425, \mathrm{n}_{\mathrm{HC}}=15, \mathrm{n}_{\mathrm{SZ}}=16, p=0.675$; week $6, \mathrm{z}=2.46$, $\left.\mathrm{n}_{\mathrm{HC}}=14, \mathrm{n}_{\mathrm{SZ}}=16, p=0.02\right)$.

\section{fMRI Results}

\section{Baseline Comparisons and Effects of Medication}

Whole brain analyses demonstrated that both $\mathrm{HC}$ and SZ displayed significant BOLD response bilaterally in the insula, dorsal and ventral prefrontal and parietal cortices, superior and middle temporal cortex, thalamus and putamen during encode correct trials. Compared to $\mathrm{HC}$, 


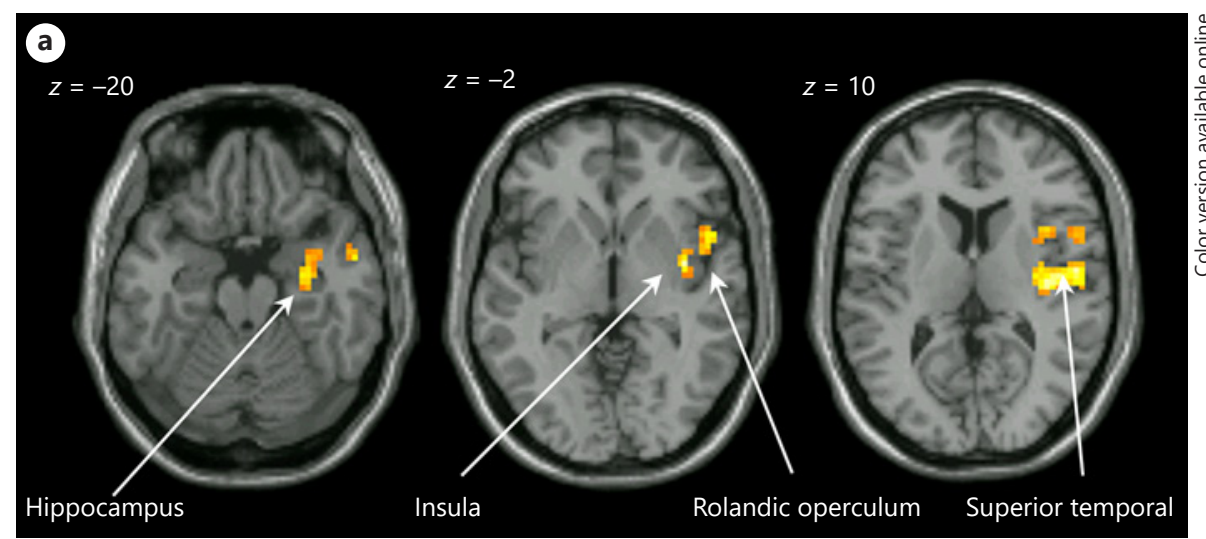

Fig. 1. Unmedicated baseline betweengroup differences during memory encoding. a Whole brain analysis: brain regions significantly more activated in HCs compared with unmedicated patients with SZ during correct memory encoding $\left(p_{\text {FDR }}<0.05\right) . \mathrm{x}, \mathrm{y}, \mathrm{z}$ refer to MNI space. b Using a DMN mask, greater BOLD response in the precuneus and the posterior cingulate were observed in SZ compared to HC. Analysis corrected using small-volume correction of $p<0.05$ ).

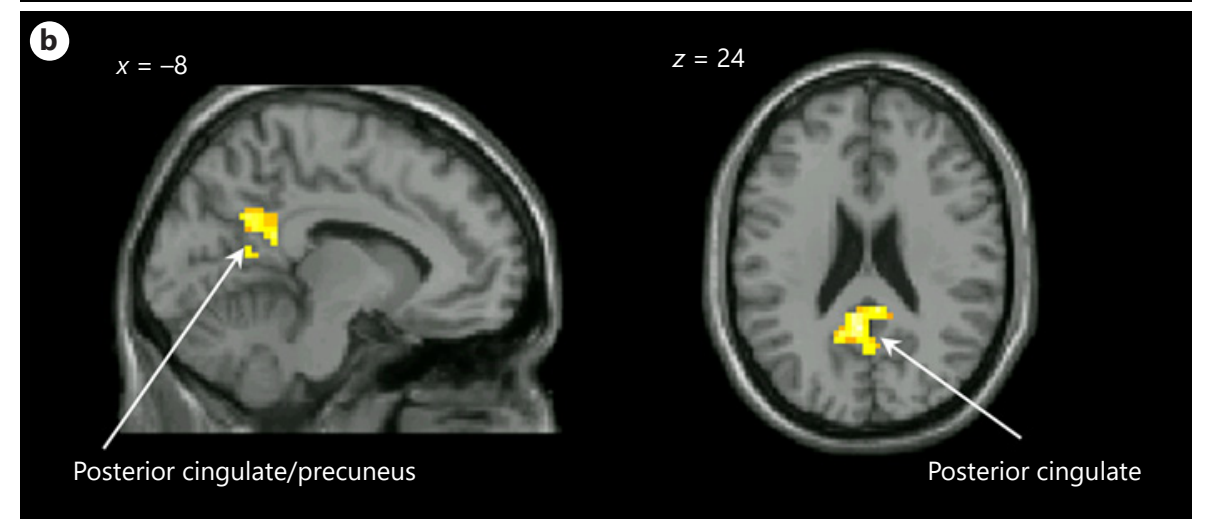

Table 2. Reduced BOLD response in unmedicated patients with SZ compared to controls

\begin{tabular}{|c|c|c|c|c|c|c|c|}
\hline \multirow[t]{2}{*}{ Cluster } & \multirow[t]{2}{*}{$\mathrm{Hem}^{\mathrm{a}}$} & \multirow[t]{2}{*}{ Voxels } & \multirow[t]{2}{*}{$z$ value } & \multicolumn{3}{|c|}{ MNI coordinates ${ }^{\mathrm{b}}$} & \multirow[t]{2}{*}{ Location $^{c}$} \\
\hline & & & & $\mathrm{x}$ & $\mathrm{y}$ & $\mathrm{z}$ & \\
\hline \multirow[t]{11}{*}{1} & $\mathrm{R}$ & 113 & 3.42 & 57 & -15 & 12 & Rolandic operculum \\
\hline & & 148 & 3.29 & 39 & -3 & 0 & Insula \\
\hline & & 83 & 3.26 & 63 & -27 & 24 & Supramarginal \\
\hline & & 60 & 3.1 & 51 & 9 & 3 & Frontal inferior \\
\hline & & 37 & 3.1 & 63 & -9 & 18 & Postcentral \\
\hline & & 21 & 2.99 & 51 & -6 & 6 & Heschl \\
\hline & & 12 & 2.64 & 48 & 0 & -30 & Temporal middle \\
\hline & & 15 & 2.53 & 51 & -6 & 3 & Temporal superior \\
\hline & & 17 & 2.36 & 30 & -9 & -21 & Hippocampus \\
\hline & & 6 & 2 & 36 & 0 & -24 & Amygdala \\
\hline & & 8 & 1.99 & 33 & 6 & -24 & Temporal pole superior \\
\hline
\end{tabular}

Cluster level FDR of 0.05 was used to control for multiple comparisons.

${ }^{a}$ Hem, hemisphere; R, right. ${ }^{b}$ Reported in MNI coordinates ( $\mathrm{x}, \mathrm{y}$, and $\mathrm{z}$ ). ${ }^{\mathrm{c}}$ Spatial localization of significant activation was defined using AAL.

BOLD, blood oxygen level dependent; SZ, schizophrenia; MNI, Montreal Neurological Institute; FDR, false discovery rate; AAL, automated anatomical labeling.

umedicated SZ revealed reduced BOLD response in the right insula, hippocampus, inferior frontal, and temporal cortex (Table 2, Fig. 1a). Using a DMN mask, greater BOLD responses (Fig. 1b) in the precuneus (peak voxel- level MNI coordinates: $\mathrm{x}=0, \mathrm{y}=-63, \mathrm{z}=27, \mathrm{z}=3.07)$ and the posterior cingulate (peak voxel-level MNI coordinates $\mathrm{x}=-3, \mathrm{y}=-51, \mathrm{z}=24, \mathrm{z}=2.89$ ) were observed in SZ compared to HC.
16

Neuropsychobiology 2021;80:12-24 DOI: $10.1159 / 000506402$
Gurler/White/Kraguljac/Ver Hoef/ Martin/Tennant/Lahti 
Fig. 2. Effects of antipsychotic medication on patterns of activation during memory encoding. A full factorial analysis revealed significant group $\times$ time interactions during encoding in the temporal and insula cortex (a), and in the parahippocampus, hippocampus, and amygdala (b; $\left.p_{\text {FDR }}<0.05\right)$. $\mathrm{x}, \mathrm{y}, \mathrm{z}$ refer to MNI space.

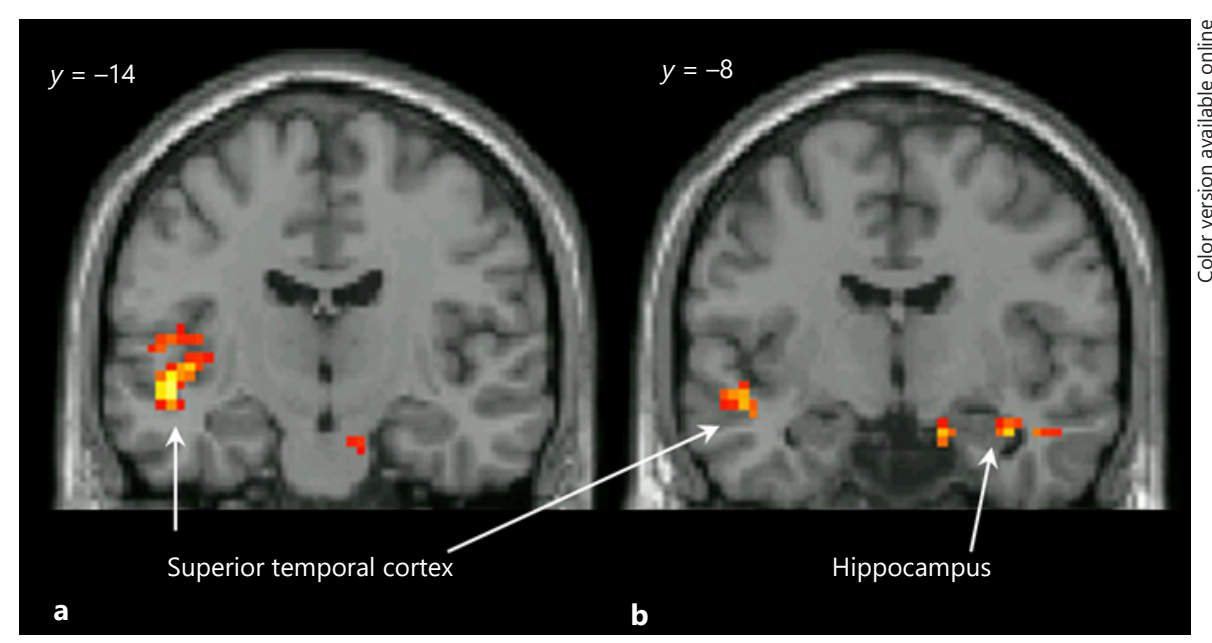

Table 3. BOLD response: significant group $\times$ time interactions

\begin{tabular}{|c|c|c|c|c|c|c|c|}
\hline \multirow[t]{2}{*}{ Cluster } & \multirow[t]{2}{*}{$\mathrm{Hem}^{\mathrm{a}}$} & \multirow[t]{2}{*}{ Voxels } & \multirow[t]{2}{*}{$z$ value } & \multicolumn{3}{|c|}{ MNI coordinates ${ }^{\mathrm{b}}$} & \multirow[t]{2}{*}{ Location } \\
\hline & & & & $\mathrm{x}$ & $\mathrm{y}$ & $\mathrm{z}$ & \\
\hline 1 & $\mathrm{~L}$ & 137 & $\begin{array}{r}3.59 \\
2.65 \\
2.2 \\
2.18\end{array}$ & $\begin{array}{l}-48 \\
-54 \\
-36 \\
-48\end{array}$ & $\begin{array}{l}-18 \\
-21 \\
-12 \\
-15\end{array}$ & $\begin{array}{r}-3 \\
-6 \\
6 \\
9\end{array}$ & $\begin{array}{l}\text { Temporal superior } \\
\text { Temporal middle } \\
\text { Insula } \\
\text { Heschl }\end{array}$ \\
\hline 2 & $\mathrm{R}$ & 128 & $\begin{array}{l}3.02 \\
2.98 \\
2.59 \\
2.33\end{array}$ & $\begin{array}{l}18 \\
36 \\
33 \\
57\end{array}$ & $\begin{array}{r}-6 \\
-6 \\
0 \\
0\end{array}$ & $\begin{array}{l}-21 \\
-24 \\
-21 \\
-21\end{array}$ & $\begin{array}{l}\text { Parahippocampal } \\
\text { Hippocampus } \\
\text { Amygdala } \\
\text { Temporal middle }\end{array}$ \\
\hline
\end{tabular}

Correction for multiple comparisons were defined by Monte Carlo simulations using a voxel level threshold at $p=0.05$ with 1,000 simulations.

${ }^{a}$ Hem, hemisphere; R, right; L, left. ${ }^{b}$ Reported in MNI coordinates (x,y, and z). ${ }^{c}$ Spatial localization of significant activation was defined using AAL.

BOLD, blood oxygen level dependent; MNI, Montreal Neurological Institute; AAL, automated anatomical labeling.

A full-factorial analysis revealed significant group $x$ time interaction in the left hemisphere in the superior and middle temporal cortex, Heschl's gyrus and insula; in the right hemisphere, in the parahippocampal gyrus, hippocampus, amygdala, and middle temporal cortex (Table 3, Fig. 2). In those regions, post hoc contrasts revealed reduced BOLD response at baseline and subsequent increase at week 6 in SZ (Fig. 3a).

To examine if these patterns impacted performance, we explored the relationship between regions where significant group $\times$ time interactions were identified and memory performance. In $\mathrm{HC}$, but not in SZ, more activation in these regions was linked to better performance.
The correlation analysis between activation in these regions and $\mathrm{d}^{\prime}$ was significantly different between $\mathrm{HC}$ and $\mathrm{SZ}$ at baseline but not at week 6 in the amygdala (baseline, $\mathrm{z}=1.75, \mathrm{n}_{\mathrm{HC}}=17, \mathrm{n}_{\mathrm{SZ}}=17, p=0.04$; week $6, \mathrm{z}=-1.36$, $\mathrm{n}_{\mathrm{HC}}=17, \mathrm{n}_{\mathrm{SZ}}=17, p=0.1$ ), and, at a trend level, in the parahippocampal gyrus (baseline, $\mathrm{z}=1.54, \mathrm{n}_{\mathrm{HC}}=17$, $\mathrm{n}_{\mathrm{SZ}}=17, p=0.06$; week $6, \mathrm{z}=-0.43, \mathrm{n}_{\mathrm{HC}}=17, \mathrm{n}_{\mathrm{SZ}}=17$, $p=0.33$; Fig. 3b).

\section{Relationship between Glutamate and the BOLD} Signal

To explore the relationship between hippocampal Glx and DMN BOLD response, we conducted a correla- 

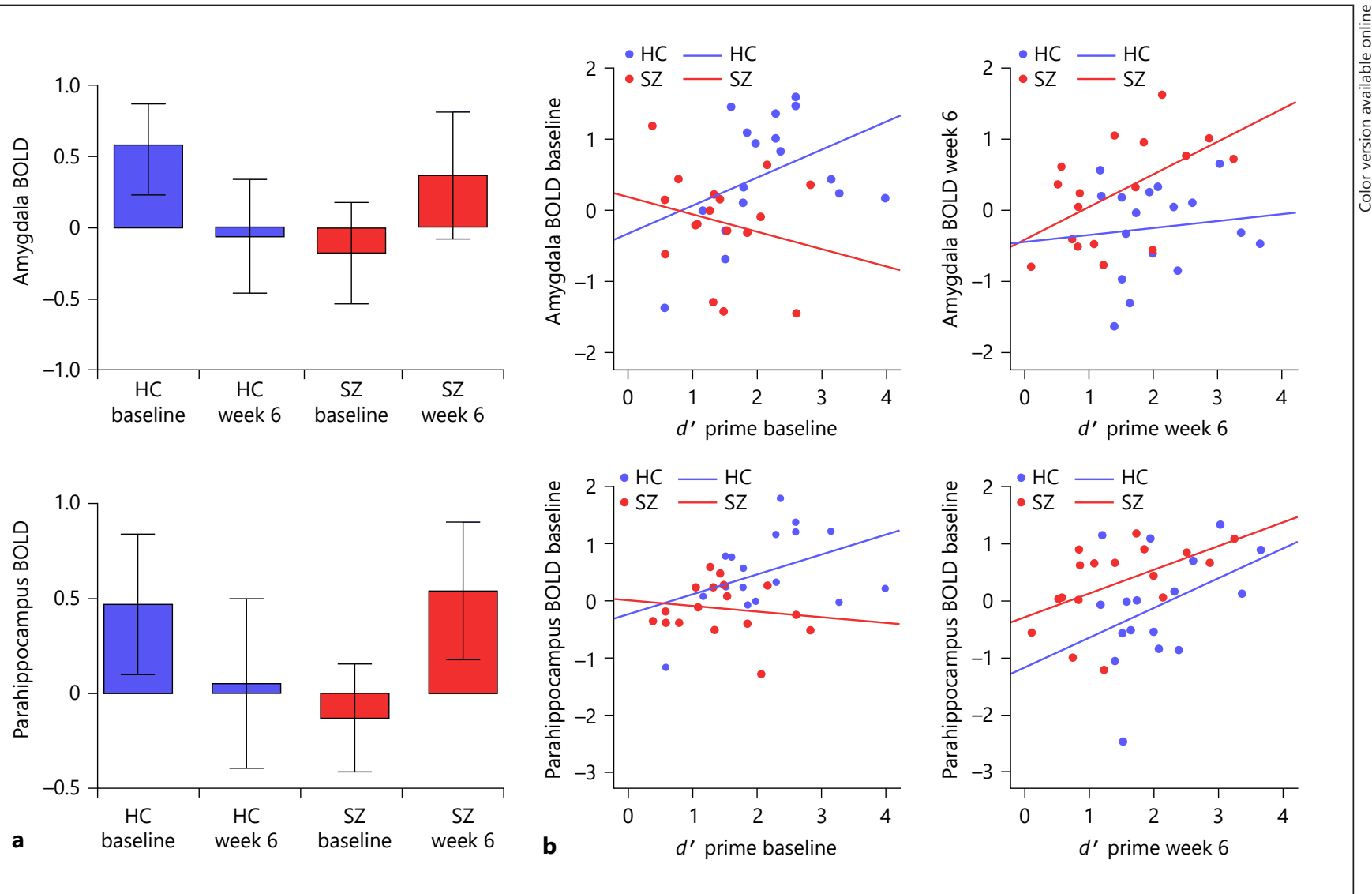

Fig. 3. a In regions where a significant group $\times$ time interaction was identified, post hoc contrasts revealed reduced BOLD response at baseline, and subsequent increase after 6 weeks in patients with SZ. b Correlations between BOLD response in these regions and memory performance using d-prime $\left(\mathrm{d}^{\prime}\right)$ were sig- nificantly different between the groups at baseline but not at week 6 in the amygdala, and, at a trend level, in the parahippocampus gyrus. BOLD, blood oxygen level dependent; HC, healthy controls; SZ, schizophrenia. tion analysis between these variables. At baseline, in SZ but not in $\mathrm{HC}$, there was a significant correlation between hippocampal Glx and BOLD response measured in the region of the posterior DMN where SZ showed increased activation $(r=0.49, n=16, p=0.03)$. The relationship between the BOLD signal in the regions of the DMN (using the full DMN mask) and hippocampal Glx was significantly different between groups $(\mathrm{z}=$ $-1.62, \mathrm{n}_{\mathrm{HC}}=15, \mathrm{n}_{\mathrm{SZ}}=16, p=0.05$ ) at baseline (Fig. 4). Thus in these regions, higher glutamate levels were related to increased activation of the DMN in SZ. At week 6, the correlation between hippocampal Glx levels and DMN was not significant and was not significantly different from $\mathrm{HC}\left(\mathrm{z}=-0.19, \mathrm{n}_{\mathrm{HC}}=14, \mathrm{n}_{\mathrm{SZ}}=16, p=0.42\right.$; Fig. 4).

\section{Discussion}

To our knowledge, this is the first study utilizing a longitudinal design together with a control group to evaluate the BOLD response during memory encoding and its relationships with hippocampal glutamate in patients with SZ before (unmedicated) and after a 6-week course of treatment with risperidone. Our findings are: (1) In unmedicated patients, presence of a reduced BOLD response in several regions, including in the hippocampus, and greater BOLD response in regions of the DMN during correct memory encoding; (2) a significant BOLD response group by time interaction in temporal cortex, bilaterally, including in the right hippocampus; but not in regions of the DMN; and (3) an abnormal BOLD modulation of the hippocampus at baseline that appears to
18

Neuropsychobiology 2021;80:12-24 DOI: $10.1159 / 000506402$
Gurler/White/Kraguljac/Ver Hoef/ Martin/Tennant/Lahti 
Fig. 4. Correlations between hippocampal Glx and BOLD response in regions of the DMN (using the full DMN mask). This relationship was significantly different between groups at baseline, but not after week 6. Glx, glutamate + glutamine; DMN, default mode network; BOLD, blood oxygenlevel dependent; HC, healthy controls; SZ, schizophrenia.

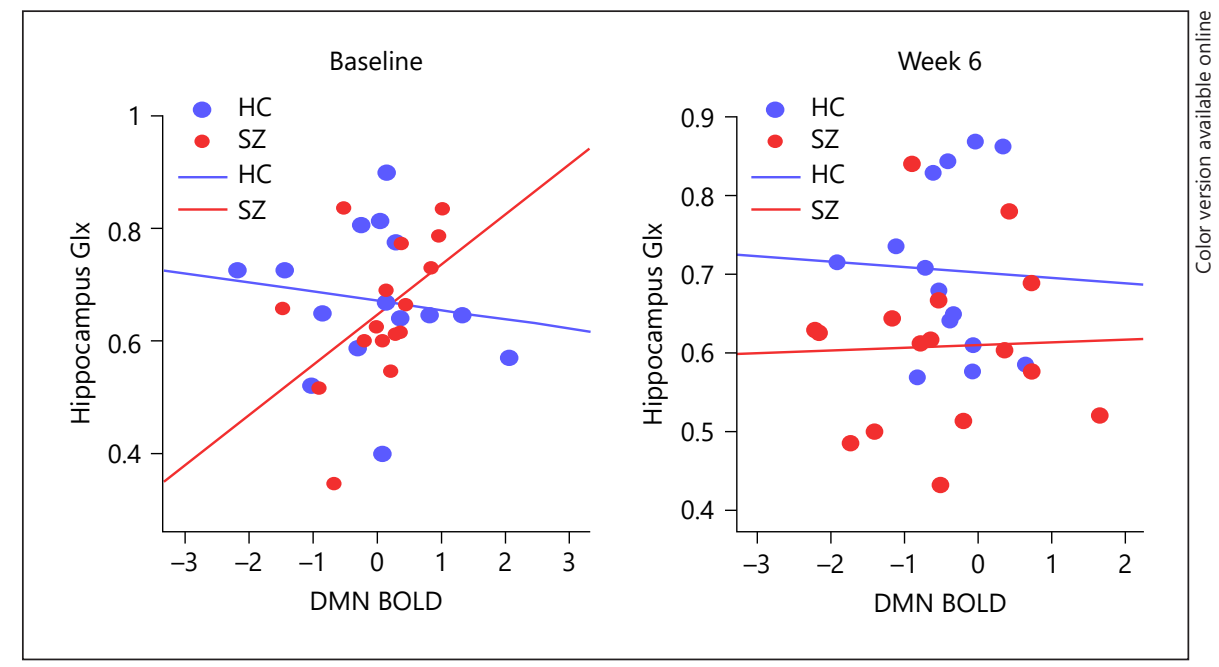

normalize at week 6; in addition, we found significant group differences in the relationship between hippocampal BOLD and performance at baseline, but not at week 6 . (4) Hippocampal Glx was not significantly different between the groups at baseline, but at week 6, hippocampal Glx was significantly lower in SZ compared to HC; (5) at baseline, higher hippocampal Glx predicted greater activation (or less deactivation) of the BOLD response in regions of the $\mathrm{DMN}$ in $\mathrm{SZ}$, but not in $\mathrm{HC}$; there were no group differences in this relationship at week 6 .

\section{Unmedicated Patterns and Effect of Medications on $B O L D$}

Hippocampal functioning in SZ has been investigated using various imaging techniques, such as PET, SPECT, arterial spin labeling, and fMRI. Abnormalities in regional cerebral blood flow [57-59], volume [60-63], and BOLD signal $[64,65]$ have been consistently reported. Given that the majority of studies enrolled medicated patients, it was important to evaluate unmedicated subjects. Here in a group of unmedicated patients, we observe hypoactivation of the hippocampus during correct memory encoding and a correlation between hippocampal BOLD and performance that was significantly different than in HC. These results are consistent with a majority of studies reporting decreased hippocampal recruitment during memory performance in SZ [65-67], including in first episode psychosis [68]. This dysfunction has also been identified in healthy siblings [23, 24], suggesting it is a heritable trait and a good candidate for intermediate phenotype. Our results in $\mathrm{HC}$ are also consistent with other studies showing that hippocampus and parahippocampus activation during encoding is predictive of subse- quent retrieval success $[18,19]$. In a group of unmedicated patients overlapping with the present group, we reported significant functional dysconnectivity between the hippocampus and other cortical regions during a resting state [69], as well as abnormal effective connectivity, measured using Granger causality methods, between bilateral hippocampi and prefrontal regions during a memory retrieval task [4].

Abnormal BOLD response in the frontal and other temporal regions in $\mathrm{SZ}$ compared to $\mathrm{HC}$ has also been reported $[64,65]$. At baseline, greater DMN BOLD response was observed in $\mathrm{SZ}$ compared to $\mathrm{HC}$ in the posterior cingulate cortex and the precuneus. Our results are consistent with previous findings of DMN hyper-activation in SZ across a variety of cognitive tasks [70-73].

We identified a significant BOLD response group by time interaction in a region encompassing the hippocampus, parahippocampus, and amygdala; in those regions, post hoc contrasts reveal reduced BOLD response at baseline with subsequent increase at week 6 , suggestive of a normalized pattern with treatment. In addition, further suggestive of a drug effect in this region, the relationship between the BOLD response and performance was no longer significantly different than that of $\mathrm{HC}$, like it was at baseline. These results are consistent with those of a previous PET study where we demonstrated a significant modulation of hippocampal regional cerebral blood flow by antipsychotic treatment during a resting state and during task performance [58]. In addition, after 1 week of antipsychotic treatment, we reported a normalization of the abnormal effective connectivity between hippocampi and prefrontal regions seen when patients were unmedicated [4]. 
Despite observing a modulation of the BOLD response in the hippocampus, we did not observe significant improvements in memory scores over time in patients. Others have reported an improvement of both BOLD response and cognition with antipsychotic medication. During a cognitive control task, medicated patients demonstrated higher dorsolateral prefrontal cortex activation as well as better behavioral performance compared to unmedicated patients [74]. Although in our study the time by group interaction did not suggest an effect of medication in regions of the DMN, in a longitudinal study, 8 weeks of antipsychotic treatment was found to improve behavioral performance and modulate the functional connectivity of the DMN in SZ during a working memory task [75]. It will be important to establish whether the changes observed in this study are associated with improvement in memory processes, as they could provide biomarkers potentially leading to the identification of novel agents for the treatment of memory impairments in SZ.

How can antipsychotic drugs improve hippocampal function? Atypical antipsychotic medications have been found to increase levels of synaptic proteins and promote dendritic growth [76]. One of these proteins, brain-derived neurotrophic factor (BDNF) which is stored and released by glutamatergic neurons, is an important regulator of synaptic transmission. BDNF is also essential to synaptic plasticity and helps protect against apoptosis $[77,78]$. Further evidence demonstrates that BDNF is associated with increases in spine density levels [79]. A large meta-analysis that included over 7,000 subjects by Fernandes et al. [80] shows that SZ is associated with lower levels of BDNF and that these levels increased with antipsychotic treatment. There is evidence that atypical antipsychotics in particular help reverse, or at least alleviate, dendritic apoptosis of the outer layers of the cortex [81].

\section{Glutamate}

At baseline, there was no significant group difference in Glx levels. We previously demonstrated elevated hippocampal Glx levels in a group of 27 unmedicated patients compared to a matched group of HC [12]. It is thus possible that our study was underpowered to show a group difference. After 6 weeks of treatment, we observed significantly lower hippocampal Glx in medicated patients compared to $\mathrm{HC}$, but not compared to their unmedicated baseline. There are a limited number of longitudinal studies evaluating the effect of short-term antipsychotic treatment on glutamatergic metabolites. In chronic patients who had undergone a medication washout, Szulc reported a decrease in temporal lobe Glx fol- lowing 4 weeks of treatment with a variety of antipsychotic medications [43]. In medication-naïve/minimally treated first episode psychosis patients, Egerton et al. [41] reported a reduction in anterior cingulate cortex glutamate after 4 weeks of treatment with amisulpride. In medication-naïve first episode psychosis subjects compared to HC, de la Fuentes-Sandoval et al. [42] observed higher baseline striatal glutamate and a significant reduction in striatal glutamate after 4 weeks of risperidone treatment. There are thus strong indications that glutamate levels are modulated by antipsychotic medications. We acknowledge that the size of our sample was limited and that there is a need to address these questions with larger sample size.

\section{Glutamate/BOLD Signal}

At baseline, higher Glx predicted less deactivation of the BOLD response in regions of the DMN in SZ, but not in $\mathrm{HC}$; this relationship was not present at week 6. In HC, $\mathrm{Hu}$ et al. [31] reported a positive relationship between high glutamate concentration in the posterior DMN and reduced DMN deactivation during a working memory task. Also in HC, Kapogiannis found a relationship between posterior DMN glutamate (posteromedial cortex) and intrinsic functional connectivity of the DMN [32]. Here, we found a correlation between hippocampal Glx and $\mathrm{BOLD}$ response in region of the DMN, a region outside that of which Glx is measured. While local neurochemical concentrations are bound to affect local neural activity, it can also be argued that they are likely to contribute to the activity of distant projections areas; this likely involves complex synaptic transmission. There now have been a number of studies demonstrating correlations between glutamate and the BOLD signal in regions distant from where glutamate was measured [33, 47, 82-84]. Because higher glutamate levels have been consistently found in medication-naïve or unmedicated SZ [10-12], one could speculate that higher Glx levels in unmedicated patients represent a pathological state altering the local ratio of excitation over inhibition and the tuning of neuronal projections with significant effects on the BOLD signal in projection areas. Intriguingly, similar to prior studies $[82,85]$, the abnormal association between BOLD response and Glx in SZ was observed in the posterior DMN. The posterior DMN have been consistently associated with successful recollection [97]; in addition, it is a major hub region that is densely interconnected with other hub regions, together forming a rich club [86]. At week 6, in the face of lower Glx levels in SZ, this association was not significant anymore. 


\section{Strengths and Limitations}

To avoid confounding medication effects and minimize data variance, we only enrolled unmedicated SZ, matched groups on several key factors, and used a rigorous longitudinal design with a single antipsychotic medication. Also, we attempted to control for the effect of time by scanning a HC group 6 weeks apart. First, we acknowledge our modest sample size; clearly replication studies will be important. This combined MRS-fMRI study obtained neurometabolite levels that, given the spectroscopy sequence in the 3T MRI scanner, were unable to distinguish the overlapping glutamate, glutamine, and GABA peaks from each other. As studies have shown differences in these metabolites in SZ [87, 88], future studies should attempt to obtain spectroscopy data from MRI scanners that are able to separate these metabolites. For fMRI analyses, we defined the DMN anatomically based on the automated anatomical labeling atlas within the Wake Forrest University Pickatlas, an automated method for neuroanatomic and cytoarchitectonic depiction of brain areas. The ROI was based on functional studies as proposed by Buckner et al. [56]. Various methods have been used to define the DMN in fMRI studies. For example, Keerativittayayut et al. [89] used both ROIs from well-established memory-related brain regions from a meta-analysis and 224 ROIs across the whole brain derived from a functional atlas [90]. Chai et al. [91] used 4 DMN seeds created around coordinates from the literature [92] to perform a group level map and identify the peaks of the group level. Perrone-Bertolotti et al. [93] used ROI analysis using a priori ROI masks for $3 \mathrm{DMN}$ regions as proposed by [92]. Sneve et al. [94] used the Yeo et al. [95] cortical parcellation to define 17 bilateral networks. The only study that used independent components analysis to highlight large-scale brain networks is the study of Santangelo et al. [96]. As can be seen, various methods have been used, and no consensus has emerged as to which method is the best. Furthermore, we performed analyses in MNI space and used DARTEL for more accurate intersubject alignment of brain images [53], but this technique does not fully account for possible individual variations in gray matter that could have affected results. Spectroscopy was done during a resting state, thus the correlations with the BOLD signal obtained during task cannot be interpreted as being causal. Further studies combining fMRI with functional MRS, where changes in neurometabolites are measured during task performance, might provide a more fine-grained understanding of the link between metabolites and cognitive processes.

Memory Encoding in SZ

\section{Conclusions}

Cognition is impaired in SZ, and to this day there is no pharmacological treatment to remediate it. It is imperative to characterize specific underlying pathologies of memory processing in the illness in order to effectively develop new treatment. Using 2 brain imaging modalities allowed us to concurrently investigate different putative mechanisms involved in memory encoding dysfunction in SZ. We confirmed that hippocampal pathology during memory encoding stems from decreased hippocampal recruitment and faulty deactivation of the DMN and that hippocampal recruitment during memory encoding is modulated by antipsychotic treatment with subsequent normalization of the relationship between BOLD and task performance. Finally, we showed that high Glx in unmedicated patients predicts less deactivation of the DMN; these results which need to be replicated with larger groups suggest a mechanism by which faulty DMN deactivation, a hallmark of pathological findings in SZ, is achieved.

\section{Acknowledgments}

This work was supported by NIMH grants R01 MH 081014 and 102951 (A.C.L.). The funding sources had no role in the study design, data collection, data analysis, manuscript preparation, or decision to publish.

\section{Statement of Ethics}

This research complies with the guidelines for human studies and was conducted in accordance with the World Medical Association Declaration of Helsinki.

\section{Disclosure Statements}

The authors have no conflicts of interest to declare.

\section{Funding Sources}

This work was supported by NIMH grants R01 MH 081014 and 102951 (A.C.L.). The funding sources had no role in the study design, data collection, data analysis, manuscript preparation, or decision to publish.

\section{Author Contributions}

A.C.L. designed the study and oversaw all aspects of the protocol. D.G. and D.M.W. undertook the data analysis. All authors contributed to interpretation of data. D.G. wrote the first draft of the manuscript. All authors contributed to and have approved the final manuscript.

Neuropsychobiology 2021;80:12-24 


\section{References}

1 Saykin AJ, Gur RC, Gur RE, Mozley PD, Mozley LH, Resnick SM, et al. Neuropsychological function in schizophrenia. Selective impairment in memory and learning. Arch Gen Psychiatry. 1991 Jul;48(7):618-24.

2 Kopald BE, Mirra KM, Egan MF, Weinberger DR, Goldberg TE. Magnitude of impact of executive functioning and IQ on episodic memory in Schizophrenia. Biol Psychiatry. 2012 Mar;71(6):545-51.

3 Sui J, Yu Q, He H, Pearlson GD, Calhoun VD. A selective review of multimodal fusion methods in schizophrenia. Front Hum Neurosci. 2012 Feb;6:27.

4 Hutcheson NL, Sreenivasan KR, Deshpande G, Reid MA, Hadley J, White DM, et al. Effective connectivity during episodic memory retrieval in schizophrenia participants before and after antipsychotic medication. Hum Brain Mapp. 2015 Apr;36(4):1442-57.

5 Ragland JD, Gur RC, Valdez J, Turetsky BI, Elliott M, Kohler C, et al. Event-related fMRI of frontotemporal activity during word encoding and recognition in schizophrenia. Am J Psychiatry. 2004 Jun;161(6):1004-15.

6 Petroff OA. GABA and glutamate in the human brain. Neuroscientist. 2002 Dec;8(6): 562-73.

7 Magistretti PJ, Pellerin L. Cellular mechanisms of brain energy metabolism. Relevance to functional brain imaging and to neurodegenerative disorders. Ann N Y Acad Sci. 1996 Jan;777(1):380-7.

8 Moffett JR, Ross B, Arun P, Madhavarao CN Namboodiri AM. N-Acetylaspartate in the CNS: from neurodiagnostics to neurobiology. Prog Neurobiol. 2007 Feb;81(2):89-131.

9 Robbins TW, Murphy ER. Behavioural pharmacology: 40+ years of progress, with a focus on glutamate receptors and cognition. Trends Pharmacol Sci. 2006 Mar;27(3):141-8.

10 de la Fuente-Sandoval C, León-Ortiz P, Favila R, Stephano S, Mamo D, Ramírez-Bermúdez J, et al. Higher levels of glutamate in the associative-striatum of subjects with prodromal symptoms of schizophrenia and patients with first-episode psychosis. Neuropsychopharmacology. 2011 Aug;36(9):1781-91.

11 Kegeles LS, Mao X, Stanford AD, Girgis R, Ojeil N, Xu X, et al. Elevated prefrontal cortex $\gamma$-aminobutyric acid and glutamate-glutamine levels in schizophrenia measured in vivo with proton magnetic resonance spectroscopy. Arch Gen Psychiatry. 2012 May;69(5): 449-59.

12 Kraguljac NV, White DM, Reid MA, Lahti AC. Increased hippocampal glutamate and volumetric deficits in unmedicated patients with schizophrenia. JAMA Psychiatry. 2013 Dec;70(12):1294-302.

13 Cairo TA, Woodward TS, Ngan ET. Decreased encoding efficiency in schizophrenia. Biol Psychiatry. 2006 Apr;59(8):740-6.
14 Cirillo MA, Seidman LJ. Verbal declarative memory dysfunction in schizophrenia: from clinical assessment to genetics and brain mechanisms. Neuropsychol Rev. 2003 Jun; 13(2):43-77.

15 Preston AR, Eichenbaum H. Interplay of hippocampus and prefrontal cortex in memory. Curr Biol. 2013 Sep;23(17):R764-73.

16 Preston AR, Shohamy D, Tamminga CA, Wagner AD. Hippocampal function, declarative memory, and schizophrenia: anatomic and functional neuroimaging considerations. Curr Neurol Neurosci Rep. 2005 Jul;5(4):249-56.

17 Shohamy D, Wagner AD. Integrating memories in the human brain: hippocampal-midbrain encoding of overlapping events. Neuron. 2008 Oct;60(2):378-89.

18 Brewer JB, Zhao Z, Desmond JE, Glover GH, Gabrieli JD. Making memories: brain activity that predicts how well visual experience will be remembered. Science. 1998 Aug;281(5380): $1185-7$.

19 Jackson O 3rd, Schacter DL. Encoding activity in anterior medial temporal lobe supports subsequent associative recognition. Neuroimage. 2004 Jan;21(1):456-62.

20 Jessen F, Scheef L, Germeshausen L, Tawo Y, Kockler M, Kuhn KU, et al. Reduced hippocampal activation during encoding and recognition of words in schizophrenia patients. Am J Psychiatry. 2003 Jul;160(7):1305-12.

21 Achim AM, Bertrand MC, Sutton H, Montoya A, Czechowska Y, Malla AK, et al. Selective abnormal modulation of hippocampal activity during memory formation in first-episode psychosis. Arch Gen Psychiatry. 2007 Sep;64(9):999-1014.

22 Goldberg TE, Torrey EF, Gold JM, Bigelow LB, Ragland RD, Taylor E, et al. Genetic risk of neuropsychological impairment in schizophrenia: a study of monozygotic twins discordant and concordant for the disorder. Schizophr Res. 1995 Sep;17(1):77-84.

23 Pirnia T, Woods RP, Hamilton LS, Lyden $\mathrm{H}$, Joshi SH, Asarnow RF, et al. Hippocampal dysfunction during declarative memory encoding in schizophrenia and effects of genetic liability. Schizophr Res. 2015 Feb;161(2-3): 357-66.

24 Rasetti R, Mattay VS, White MG, Sambataro F, Podell JE, Zoltick B, et al. Altered hippocampal-parahippocampal function during stimulus encoding: a potential indicator of genetic liability for schizophrenia. JAMA Psychiatry. 2014 Mar;71(3):236-47.

25 Kraguljac NV, Srivastava A, Lahti AC. Memory deficits in schizophrenia: a selective review of functional magnetic resonance imaging (FMRI) studies. Behav Sci (Basel). 2013 Jun;3(3):330-47.

26 Anticevic A, Repovs G, Shulman GL, Barch DM. When less is more: TPJ and default network deactivation during encoding predicts working memory performance. Neuroimage. 2010 Feb;49(3):2638-48.
27 Raichle ME. The brain's default mode network. Annu Rev Neurosci. 2015 Jul;38(1): 433-47.

28 Anticevic A, Repovs G, Barch DM. Working memory encoding and maintenance deficits in schizophrenia: neural evidence for activation and deactivation abnormalities. Schizophr Bull. 2013 Jan;39(1):168-78.

29 Whitfield-Gabrieli S, Thermenos HW, Milanovic S, Tsuang MT, Faraone SV, McCarley RW, et al. Hyperactivity and hyperconnectivity of the default network in schizophrenia and in first-degree relatives of persons with schizophrenia. Proc Natl Acad Sci USA. 2009 Jan;106(4):1279-84.

30 Shulman RG, Rothman DL, Behar KL, Hyder F. Energetic basis of brain activity: implications for neuroimaging. Trends Neurosci. 2004 Aug;27(8):489-95.

$31 \mathrm{Hu}$ Y, Chen X, Gu H, Yang Y. Resting-state glutamate and GABA concentrations predict taskinduced deactivation in the default mode network. J Neurosci. 2013 Nov;33(47):18566-73.

32 Kapogiannis D, Reiter DA, Willette AA, Mattson MP. Posteromedial cortex glutamate and GABA predict intrinsic functional connectivity of the default mode network. Neuroimage. 2013 Jan;64:112-9.

33 Falkenberg LE, Westerhausen R, Craven AR, Johnsen E, Kroken RA, L Berg EM, et al. Impact of glutamate levels on neuronal response and cognitive abilities in schizophrenia. Neuroimage Clin. 2014 Apr;4:576-84.

34 Overbeek G. Relationship between glutamate and BOLD Stroop effect in First-Episode schizophrenia. Manuscript Submitted 2018.

35 Lahti AC, Weiler MA, Holcomb HH, Tamminga CA, Cropsey KL. Modulation of limbic circuitry predicts treatment response to antipsychotic medication: a functional imaging study in schizophrenia. Neuropsychopharmacology. 2009 Dec;34(13):2675-90.

36 Kraguljac NV, White DM, Hadley JA, Visscher K, Knight D, ver Hoef L, et al. Abnormalities in large scale functional networks in unmedicated patients with schizophrenia and effects of risperidone. Neuroimage Clin. 2015 Nov; 10:146-58.

37 HoneyGD, BullmoreET, Soni W, Varatheesan M, Williams SC, Sharma T. Differences in frontal cortical activation by a working memory task after substitution of risperidone for typical antipsychotic drugs in patients with schizophrenia. Proc Natl Acad Sci USA. 1999 Nov;96(23):13432-7.

38 Minzenberg MJ, Carter CS. Developing treatments for impaired cognition in schizophrenia. Trends Cogn Sci. 2012 Jan;16(1):35-42.

39 Keefe RS, Bilder RM, Davis SM, Harvey PD, Palmer BW, Gold JM, et al.; CATIE Investigators; Neurocognitive Working Group. Neurocognitive effects of antipsychotic medications in patients with chronic schizophrenia in the CATIE Trial. Arch Gen Psychiatry. 2007 Jun;64(6):633-47. 
40 Davidson M, Galderisi S, Weiser M, Werbeloff N, Fleischhacker WW, Keefe RS, et al. Cognitive effects of antipsychotic drugs in first-episode schizophrenia and schizophreniform disorder: a randomized, open-label clinical trial (EUFEST). Am J Psychiatry. 2009 Jun;166(6):675-82.

41 Egerton A, Broberg BV, Van Haren N, Merritt K, Barker GJ, Lythgoe DJ, et al. Response to initial antipsychotic treatment in first episode psychosis is related to anterior cingulate glutamate levels: a multicentre $1 \mathrm{H}-\mathrm{MRS}$ study (OPTiMiSE). Mol Psychiatry. 2018 Nov; 23(11):2145-55.

42 de la Fuente-Sandoval C, León-Ortiz P, Azcárraga M, Stephano S, Favila R, Díaz-Galvis $\mathrm{L}$, et al. Glutamate levels in the associative striatum before and after 4 weeks of antipsychotic treatment in first-episode psychosis: a longitudinal proton magnetic resonance spectroscopy study. JAMA Psychiatry. 2013 Oct;70(10):1057-66.

43 Szulc A, Galinska B, Tarasow E, Waszkiewicz N, Konarzewska B, Poplawska R, et al. Proton magnetic resonance spectroscopy study of brain metabolite changes after antipsychotic treatment. Pharmacopsychiatry. 2011 Jun; 44(4):148-57.

44 Nurnberger JI Jr, Blehar MC, Kaufmann CA, York-Cooler C, Simpson SG, Harkavy-Friedman J, et al.; NIMH Genetics Initiative. Diagnostic interview for genetic studies. Rationale, unique features, and training. Arch Gen Psychiatry. 1994 Nov;51(11):849-59.

45 Randolph C, Tierney MC, Mohr E, Chase TN. The repeatable battery for the assessment of neuropsychological status (RBANS): preliminary clinical validity. J Clin Exp Neuropsychol. 1998 Jun;20(3):310-9.

46 Overall JE, Gorham DE. The Brief Psychiatric Rating Scale. Psychol Rep. 1962;10(3):799812.

47 Hutcheson NL, Reid MA, White DM, Kraguljac NV, Avsar KB, Bolding MS, et al. Multimodal analysis of the hippocampus in schizophrenia using proton magnetic resonance spectroscopy and functional magnetic resonance imaging. Schizophr Res. 2012 Sep; 140(1-3):136-42.

48 Stanislaw $\mathrm{H}$, Todorov N. Calculation of signal detection theory measures. Behav Res Methods Instrum Comput. 1999 Feb;31(1):137-49.

49 Schubert F, Gallinat J, Seifert F, Rinneberg H. Glutamate concentrations in human brain using single voxel proton magnetic resonance spectroscopy at 3 Tesla. Neuroimage. 2004 Apr;21(4):1762-71.

50 Vanhamme L, van den Boogaart A, Van Huffel S. Improved method for accurate and efficient quantification of MRS data with use of prior knowledge. J Magn Reson. 1997 Nov; 129(1):35-43.

51 Reid MA, Stoeckel LE, White DM, Avsar KB, Bolding MS, Akella NS, et al. Assessments of function and biochemistry of the anterior cingulate cortex in schizophrenia. Biol Psychiatry. 2010 Oct;68(7):625-33.
52 Ratiney H, Coenradie Y, Cavassila S, van Ormondt D, Graveron-Demilly D. Time-domain quantitation of $1 \mathrm{H}$ short echo-time signals: background accommodation. MAGMA. 2004 May;16(6):284-96.

53 Ashburner J. A fast diffeomorphic image registration algorithm. Neuroimage. 2007 Oct; 38(1):95-113.

54 Chumbley JR, Friston KJ. False discovery rate revisited: FDR and topological inference using Gaussian random fields. Neuroimage. 2009 Jan;44(1):62-70.

55 Maldjian JA, Laurienti PJ, Kraft RA, Burdette $\mathrm{JH}$. An automated method for neuroanatomic and cytoarchitectonic atlas-based interrogation of fMRI data sets. Neuroimage. 2003 Jul;19(3):1233-9.

56 Buckner RL, Andrews-Hanna JR, Schacter DL. The brain's default network: anatomy, function, and relevance to disease. Ann N Y Acad Sci. 2008 Mar;1124(1):1-38.

57 Heckers S, Rauch SL, Goff D, Savage CR, Schacter DL, Fischman AJ, et al. Impaired recruitment of the hippocampus during conscious recollection in schizophrenia. Nat Neurosci. 1998 Aug;1(4):318-23.

58 Medoff DR, Holcomb HH, Lahti AC, Tamminga CA. Probing the human hippocampus using rCBF: contrasts in schizophrenia. Hippocampus. 2001;11(5):543-50.

59 Pinkham A, Loughead J, Ruparel K, Wu WC, Overton E, Gur R, et al. Resting quantitative cerebral blood flow in schizophrenia measured by pulsed arterial spin labeling perfusion MRI. Psychiatry Res. 2011 Oct;194(1): 64-72.

60 Schobel SA, Lewandowski NM, Corcoran CM, Moore H, Brown T, Malaspina D, et al. Differential targeting of the CA1 subfield of the hippocampal formation by schizophrenia and related psychotic disorders. Arch Gen Psychiatry. 2009 Sep;66(9):938-46.

61 Schobel SA, Chaudhury NH, Khan UA, Paniagua B, Styner MA, Asllani I, et al. Imaging patients with psychosis and a mouse model establishes a spreading pattern of hippocampal dysfunction and implicates glutamate as a driver. Neuron. 2013 Apr;78(1):81-93.

62 Talati P, Rane S, Kose S, Blackford JU, Gore J, Donahue MJ, et al. Increased hippocampal CA1 cerebral blood volume in schizophrenia. Neuroimage Clin. 2014 Jul;5:359-64.

63 Talati P, Rane S, Skinner J, Gore J, Heckers S. Increased hippocampal blood volume and normal blood flow in schizophrenia. Psychiatry Res. 2015 Jun;232(3):219-25.

64 Ragland JD, Laird AR, Ranganath C, Blumenfeld RS, Gonzales SM, Glahn DC. Prefrontal activation deficits during episodic memory in schizophrenia. Am J Psychiatry. 2009 Aug; 166(8):863-74.

65 Achim AM, Lepage M. Episodic memory-related activation in schizophrenia: meta-analysis. Br J Psychiatry. 2005 Dec;187(6):500-9.
66 Ragland JD, Ranganath C, Harms MP, Barch DM, Gold JM, Layher E, et al. Functional and Neuroanatomic Specificity of Episodic Memory Dysfunction in Schizophrenia: A Functional Magnetic Resonance Imaging Study of the Relational and Item-Specific Encoding Task. JAMA Psychiatry. 2015 Sep;72(9):909-16.

67 Leube DT, Rapp A, Buchkremer G, Bartels M, Kircher TT, Erb M, et al. Hippocampal dysfunction during episodic memory encoding in patients with schizophrenia-an fMRI study. Schizophr Res. 2003 Nov;64(1):83-5.

68 Francis MM, Hummer TA, Vohs JL, Yung MG, Liffick E, Mehdiyoun NF, et al. Functional neuroanatomical correlates of episodic memory impairment in early phase psychosis. Brain Imaging Behav. 2016 Mar;10(1):1-11.

69 Kraguljac NV, White DM, Hadley N, Hadley JA, Ver Hoef L, Davis E, et al. Aberrant Hippocampal Connectivity in Unmedicated $\mathrm{Pa}$ tients With Schizophrenia and Effects of Antipsychotic Medication: A Longitudinal Resting State Functional MRI Study. Schizophr Bull. 2016 Jul;42(4):1046-55.

70 Garrity AG, Pearlson GD, McKiernan K, Lloyd D, Kiehl KA, Calhoun VD. Aberrant "default mode" functional connectivity in schizophrenia. Am J Psychiatry. 2007 Mar; 164(3):450-7.

71 Ongür D, Lundy M, Greenhouse I, Shinn AK, Menon V, Cohen BM, et al. Default mode network abnormalities in bipolar disorder and schizophrenia. Psychiatry Res. 2010 Jul; 183(1):59-68.

72 Pomarol-Clotet E, Salvador R, Sarró S, Gomar J, Vila F, Martínez A, et al. Failure to deactivate in the prefrontal cortex in schizophrenia: dysfunction of the default mode network? Psychol Med. 2008 Aug;38(8):1185-93.

73 Salgado-Pineda P, Fakra E, Delaveau P, McKenna PJ, Pomarol-Clotet E, Blin O. Correlated structural and functional brain abnormalities in the default mode network in schizophrenia patients. Schizophr Res. 2011 Feb;125(2-3):101-9.

74 Lesh TA, Tanase C, Geib BR, Niendam TA, Yoon JH, Minzenberg MJ, et al. A multimodal analysis of antipsychotic effects on brain structure and function in first-episode schizophrenia. JAMA Psychiatry. 2015 Mar;72(3): 226-34.

75 Sambataro F, Blasi G, Fazio L, Caforio G, Taurisano $\mathrm{P}$, Romano $\mathrm{R}$, et al. Treatment with olanzapine is associated with modulation of the default mode network in patients with Schizophrenia. Neuropsychopharmacology. 2010 Mar;35(4):904-12.

76 Park SW, Lee CH, Cho HY, Seo MK, Lee JG, Lee BJ, et al. Effects of antipsychotic drugs on the expression of synaptic proteins and dendritic outgrowth in hippocampal neuronal cultures. Synapse. 2013 May;67(5):224-34.

77 Kuipers SD, Bramham CR. Brain-derived neurotrophic factor mechanisms and function in adult synaptic plasticity: new insights and implications for therapy. Curr Opin Drug Discov Devel. 2006 Sep;9(5):580-6. 
78 Pandya CD, Kutiyanawalla A, Pillai A. BDNFTrkB signaling and neuroprotection in schizophrenia. Asian J Psychiatr. 2013 Feb; 6(1):22-8

79 Tyler WJ, Pozzo-Miller L. Miniature synaptic transmission and BDNF modulate dendritic spine growth and form in rat CA1 neurones. J Physiol. 2003 Dec;553(Pt 2):497-509.

80 Fernandes BS, Steiner J, Berk M, Molendijk ML, Gonzalez-Pinto A, Turck CW, et al. Peripheral brain-derived neurotrophic factor in schizophrenia and the role of antipsychotics: meta-analysis and implications. Mol Psychiatry. 2015 Sep;20(9):1108-19.

81 Lieberman JA, Bymaster FP, Meltzer HY, Deutch AY, Duncan GE, Marx CE, et al. Antipsychotic drugs: comparison in animal models of efficacy, neurotransmitter regulation, and neuroprotection. Pharmacol Rev. 2008 Sep;60(3):358-403.

82 Falkenberg LE, Westerhausen R, Specht K, Hugdahl K. Resting-state glutamate level in the anterior cingulate predicts blood-oxygen level-dependent response to cognitive control. Proc Natl Acad Sci USA. 2012 Mar; 109(13):5069-73.

83 Duncan NW, Enzi B, Wiebking C, Northoff G. Involvement of glutamate in rest-stimulus interaction between perigenual and supragenual anterior cingulate cortex: a combined fMRI-MRS study. Hum Brain Mapp. 2011 Dec;32(12):2172-82.
84 White DM, Kraguljac NV, Reid MA, Lahti AC. Contribution of substantia nigra glutamate to prediction error signals in schizophrenia: a combined magnetic resonance spectroscopy/functional imaging study. NPJ Schizophr. 2015;1(1):14001.

85 Overbeek G, Gawne TJ, Reid MA, Salibi N, Kraguljac NV, White DM, et al. Relationship Between Cortical Excitation and Inhibition and Task-Induced Activation and Deactivation: A Combined Magnetic Resonance Spectroscopy and Functional Magnetic Resonance Imaging Study at 7T in First-Episode Psychosis. Biol Psychiatry Cogn Neurosci Neuroimaging. $2019 \mathrm{Feb} ; 4(2): 121-30$.

86 van den Heuvel MP, Sporns O. Rich-club organization of the human connectome. J Neurosci. 2011 Nov;31(44):15775-86.

87 Rowland LM, Kontson K, West J, Edden RA, Zhu $\mathrm{H}$, Wijtenburg SA, et al. In vivo measurements of glutamate, GABA, and NAAG in schizophrenia. Schizophr Bull. 2013 Sep; 39(5):1096-104.

88 Marsman A, Mandl RC, Klomp DW, Bohlken MM, Boer VO, Andreychenko A, et al. GABA and glutamate in schizophrenia: a $7 \mathrm{~T}^{1} \mathrm{H}$-MRS study. Neuroimage Clin. 2014 Oct;6:398-407.

89 Keerativittayayut R, Aoki R, Sarabi MT, Jimura K, Nakahara K. Large-scale network integration in the human brain tracks temporal fluctuations in memory encoding performance. eLife. 2018 Jun; 7:7.

90 Power JD, Cohen AL, Nelson SM, Wig GS, Barnes KA, Church JA, et al. Functional network organization of the human brain. Neuron. 2011 Nov;72(4):665-78.
91 Chai XJ, Ofen N, Gabrieli JD, Whitfield-Gabrieli S. Development of deactivation of the default-mode network during episodic memory formation. Neuroimage. 2014 Jan;84: 932-8.

92 Fox MD, Corbetta M, Snyder AZ, Vincent JL, Raichle ME. Spontaneous neuronal activity distinguishes human dorsal and ventral attention systems. Proc Natl Acad Sci USA. 2006 Jun;103(26):10046-51.

93 Perrone-Bertolotti M, Cerles M, Ramdeen KT, Boudiaf N, Pichat C, Hot P, et al. The SelfPleasantness Judgment Modulates the Encoding Performance and the Default Mode Network Activity. Front Hum Neurosci. 2016 Mar;10:121.

94 Sneve MH, Grydeland H, Amlien IK, Langnes E, Walhovd KB, Fjell AM. Decoupling of large-scale brain networks supports the consolidation of durable episodic memories. Neuroimage. 2017 Jun;153:336-45.

95 Yeo BT, Krienen FM, Sepulcre J, Sabuncu MR, Lashkari D, Hollinshead M, et al. The organization of the human cerebral cortex estimated by intrinsic functional connectivity. J Neurophysiol. 2011 Sep;106(3):1125-65.

96 Santangelo V, Bordier C. Large-Scale Brain Networks Underlying Successful and Unsuccessful Encoding, Maintenance, and Retrieval of Everyday Scenes in Visuospatial Working Memory. Front Psychol. 2019 Feb;10:233.

97 Vincent JL, Kahn I, Snyder AZ, Raichle ME, Buckner RL. Evidence for a frontoparietal control system revealed by intrinsic functional connectivity. J Neurophysiol. 2008 Dec; 100(6):3328-42. 